\title{
Perioperative management of antiplatelet treatment in patients undergoing isolated coronary artery bypass grafting in Dutch cardiothoracic centres
}

\author{
P. W. A. Janssen ${ }^{1,2} \cdot$ D. M. F. Claassens ${ }^{1,2} \cdot$ L. M. Willemsen ${ }^{1,2} \cdot$ T. O. Bergmeijer ${ }^{1,2} \cdot$ P. Klein $^{3} \cdot$ J. M. ten Berg ${ }^{1,2}$
}

Published online: 13 June 2017

(C) The Author(s) 2017. This article is an open access publication.

\begin{abstract}
Background International guidelines do not provide uniform recommendations regarding the use of antiplatelet treatment in the perioperative period in patients undergoing coronary artery bypass grafting (CABG).

Methods A questionnaire was sent to all 16 cardiothoracic centres in the Netherlands to determine which antiplatelet treatment is used in the perioperative setting. Furthermore, a single-centre prospective observational cohort study was performed which included all patients undergoing isolated CABG in July 2014.

Results Eleven centres responded to the survey. Acetylsalicylic acid monotherapy was discontinued before surgery in 6 centres. In patients with an acute coronary syndrome receiving dual antiplatelet therapy (DAPT), most centres discontinued the $\mathrm{P} 2 \mathrm{Y}_{12}$ inhibitor preoperatively. DAPT was restarted after surgery in 4 centres. However, 6 centres continued DAPT in patients who had undergone coronary stenting within one month of surgery. In patients with coronary stents, variation in the management of antiplatelet therapy increased in proportion to the interval between stenting and surgery. A total of 70 patients were included in the registry.
\end{abstract}

Electronic supplementary material The online version of this article (doi: 10.1007/s12471-017-1006-z) contains supplementary material, which is available to authorized users.

P. W. A. Janssen

p.janssen@antoniusziekenhuis.nl

1 St. Antonius Center for Platelet Function Research, Nieuwegein, The Netherlands

2 Department of Cardiology, St. Antonius Hospital, Nieuwegein, The Netherlands

3 Department of Cardiothoracic Surgery, St. Antonius Hospital, Nieuwegein, The Netherlands
Acetylsalicylic acid monotherapy was discontinued in 51\% of patients and restarted in all patients. $\mathrm{P} 2 \mathrm{Y}_{12}$ inhibitor treatment was discontinued before surgery in $70 \%$ of patients and re-initiated after CABG in $29 \%$.

Conclusions Major differences were observed in the preoperative and postoperative management of antiplatelet treatment between different Dutch cardiothoracic centres and within a single centre. Part of this variation is probably due to lack of evidence and differences between the current guidelines; however, many of the strategies were not in accordance with any of these guidelines.

Keywords Antiplatelet treatment $\cdot \mathrm{P} \mathrm{Y}_{12}$ inhibitor . CABG $\cdot$ Acute coronary syndrome $\cdot$ Clopidogrel · Acetylsalicylic acid

\section{Introduction}

Most patients scheduled to undergo coronary arterial bypass grafting $(\mathrm{CABG})$ are treated with acetylsalicylic acid (ASA) with or without a $\mathrm{P}_{2} \mathrm{Y}_{12}$ inhibitor (clopidogrel, prasugrel, ticagrelor) before surgery. The current guidelines from the American College of Cardiology (ACC)/American Heart Association (AHA), European Society of Cardiology (ESC) and European Association for Cardio-Thoracic Surgery (EACTS) provide different recommendations regarding the continuation or (temporary) cessation of antiplatelet drugs during the perioperative period [1-4]. In general, it is recommended to continue ASA during and after CABG [1-4]. However, some guidelines state that it can be reasonable to discontinue ASA several days before CABG in patients with stable coronary heart disease [2, 3]. The guidelines are consistent in their advice to discontinue $\mathrm{P}_{2} \mathrm{Y}_{12}$ inhibitors before surgery in stable patients 
Table 1 Preoperative management of antiplatelet therapy

\begin{tabular}{|c|c|c|c|c|}
\hline \multicolumn{5}{|c|}{ Number of centres following each strategy } \\
\hline & $\begin{array}{l}\text { ASA monotherapy } \\
\text { (N) }\end{array}$ & $\begin{array}{l}\text { DAPT Clopidogrel } \\
\text { (N) }\end{array}$ & $\begin{array}{l}\text { DAPT Prasugrel } \\
\text { (N) }\end{array}$ & $\begin{array}{l}\text { DAPT Ticagrelor } \\
\text { (N) }\end{array}$ \\
\hline Continue: & 5 & - & - & - \\
\hline \multicolumn{5}{|c|}{ Discontinue: } \\
\hline 1 day & - & - & - & - \\
\hline 2 days & - & - & - & 1 \\
\hline 3 days & 1 & - & - & - \\
\hline 4 days & 1 & 1 & 1 & 1 \\
\hline $3-5$ days & 1 & - & - & - \\
\hline 5 days & 2 & 1 & 3 & 5 \\
\hline 5-7 days & - & - & - & 1 \\
\hline 7 days & - & 1 & 2 & 1 \\
\hline $7-10$ days & 1 & 6 & 1 & - \\
\hline
\end{tabular}

The preoperative management of patients on ASA monotherapy and patients on DAPT with clopidogrel, prasugrel or ticagrelor

$A S A$ acetylsalicylic acid, DAPT dual antiplatelet therapy, $N$ number of centres without recent coronary stent implantation, although there is no consistency regarding the timing of discontinuation. In high-risk groups, i. e. patients who have recently undergone coronary stent implantation [2] or patients with a high risk for thrombotic events [4], it is recommended not to interrupt dual antiplatelet therapy (DAPT) treatment. Physicians have to decide whether the increased risk of bleeding with continued antiplatelet therapy outweighs the risk of thrombotic events associated with the discontinuation of these drugs before CABG. The use of DAPT after CABG in patients who recently experienced an acute coronary syndrome (ACS) is also a subject of debate [5]. Recent reports have shown that treatment is not re-initiated after surgery in a large portion of patients [6], despite the fact that reinitiation is recommended in the guidelines [3, 4].

We aimed to describe the use of antiplatelet treatment in the perioperative period in patients undergoing CABG in contemporary practice in the Netherlands.

\section{Materials and methods}

First, a survey was sent to all 16 centres in the Netherlands in which CABG surgery is performed. The head of the department of each centre was contacted. The survey consisted of both open and closed questions so that respondents could indicate how predefined groups of patients would be treated in general (i. e. mono antiplatelet therapy versus DAPT, patients after ACS and/or recent stent implantation) and which patients were exempted from standard treatment protocols. The survey is shown online as Electronic Supplementary Material.

Second, we conducted a prospective, observational pilot study in the St. Antonius Hospital in Nieuwegein.
All patients undergoing isolated CABG in July 2014 were included in this registry. There were no exclusion criteria. Baseline data, antiplatelet treatment and postoperative complications (with 30 days of follow-up) were registered in all patients. The study was conducted according to the principles of the Declaration of Helsinki and received approval from the local Human Research Ethics Committee. The local ethics committee provided a waiver for written informed consent, as the study was not associated with any risk.

\section{Results}

\section{Survey}

Between November 2014 and April 2015, 11 out of the16 Dutch centres in which cardiothoracic surgery is performed, including our own centre, responded to the questionnaire. The other centres are listed in the acknowledgements.

Six out of 11 centres answered that ASA monotherapy was routinely discontinued before $\mathrm{CABG}$, while 5 centres always continued ASA monotherapy (Table 1). Fig. 1a to 1c show the perioperative management of antiplatelet treatment in patients with ACS undergoing CABG during the index admission, between the index admission and 1 month after the ACS or between 1 month and 12 months after the ACS. Preoperative management differed slightly between centres, but postoperative management was the same for the different groups. Fig. $2 \mathrm{a}$ to $2 \mathrm{c}$ show the perioperative management of antiplatelet treatment in patients undergoing $\mathrm{CABG}$ within 1 month, between 1 and 6 months and between 6 and 12 months after coronary stent implantation. 


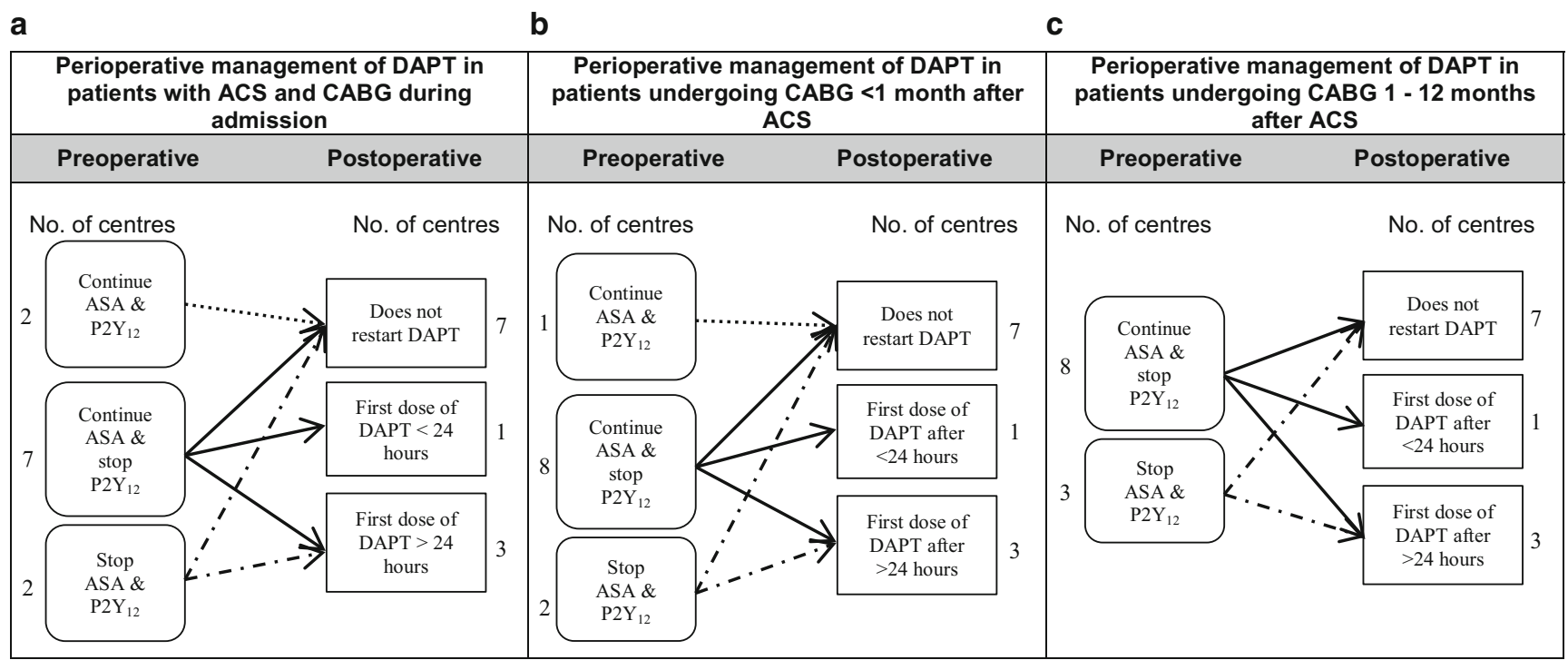

Fig. 1a-c Perioperative management of DAPT in patients with ACS and CABG during the same admission, CABG $<1$ month and CABG 1-12 months after ACS. ACS acute coronary syndrome, $A S A$ acetylsalicylic acid, $C A B G$ coronary artery bypass grafting, $D A P T$ dual antiplatelet therapy, No number, $P 2 Y_{12} \mathrm{P}_{2} \mathrm{Y}_{12}$ inhibitor

a

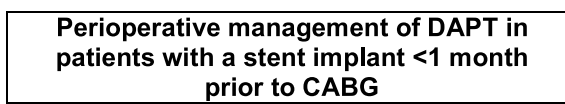
prior to CABG

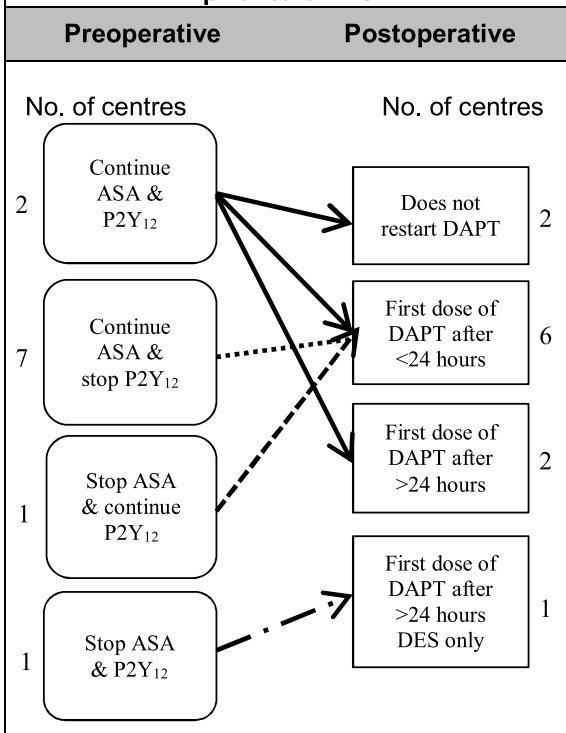

b Perioperative management of DAPT in
patients with a stent $1-6$ months prior to
CABG

Preoperative Postoperative

\begin{tabular}{l|l|l} 
No. of centres No. of centres & No. of centres No. of centres
\end{tabular}

C Perioperative management of DAPT in patients with a stent $6-12$ months prior to

\begin{tabular}{|cc} 
Preoperative & Postoperative \\
No. of centres & No. of centres
\end{tabular}

Fig. 2a-c Perioperative management of DAPT in patients undergoing CABG less than 1 month, between 1-6 months and between 6-12 months after stent implantation. $A S A$ acetylsalicylic acid, $B M S$ bare metal stent, $C A B G$ coronary artery bypass grafting, $D A P T$ dual antiplatelet therapy, $D E S$ drug-eluting stent, $I C W$ in consultation with, No number, $P 2 Y_{12} \mathrm{P}_{2} \mathrm{Y}_{12}$ inhibitor 
Table 2 Baseline characteristics

\begin{tabular}{ll}
\hline Characteristics & $N=70$ \\
& $N(\%)$
\end{tabular}

Patient characteristics

Male

Age, mean, (SD), years

$65.5 \pm 10.1$

Body mass index, mean, (SD)

$28.0 \pm 3.2$

Current smoker

$14(20.3)$

Ex-smoker ( $>6$ weeks)

$19(27.5)$

Family history for CAD

Medical history

$\begin{array}{lr}\text { Hypertension } & 58(82.9)\end{array}$

Diabetes mellitus $\quad 21(30.0)$

$\begin{array}{ll}\text { Dyslipidaemia } & 37 \text { (52.9) }\end{array}$

$\begin{array}{ll}\text { Angina pectoris month prior to surgery* } & 50(71.4)\end{array}$

$\begin{array}{ll}\text { TIA/Stroke } & 6(8.6)\end{array}$

COPD $6(8.6)$

Chronic kidney disease (eGFR MDRD4 <60 ml/min) 3 (4.3)

Peripheral arterial disease $3(4.3)$

Heart failure (NYHA class III or IV) 9 (12.9)

ACS 39 (55.7)

- MI 34 (48.6)

$\begin{array}{ll}\text { Prior PCI } & 18(26.1)\end{array}$

- PCI + Stent $11(15.7)$

$\begin{array}{ll}\text { Prior CABG } & 0(0.0)\end{array}$

Preoperative medication use

Oral nitrates 15 (21.4)

$\begin{array}{lr}\text { Beta-blockers } & 57(81.4)\end{array}$

$\begin{array}{ll}\text { ACE inhibitor } & 38(54.3)\end{array}$

$\begin{array}{lr}\text { AT-II-antagonists } & 17(24.3)\end{array}$

Diuretics 18 (25.7)

$\begin{array}{ll}\text { Statins and other lipid-lowering drugs } & 67 \text { (95.7) }\end{array}$

$\begin{array}{ll}\text { Oral antidiabetics } & 16(22.9)\end{array}$

Insulin 8 (11.4)

Surgery

Coronary artery disease

- One vessel $10(14.3)$

- Two vessel 9 (12.9)

$\begin{array}{ll}\text { - Three vessel } & 51(72.9)\end{array}$

Timing

- Elective/planned $\quad 66$ (94.3)

- Urgent 2 (2.9)

- Emergency $2(2.9)$

$\begin{array}{lr}\text { EuroScore (SD) } & 3.2 \pm 2.6\end{array}$

Data are presented as number and percentage unless otherwise indicated. Denominators to derive percentages are based on available data for each characteristic. *Any Canadian Cardiovascular Society class angina $A C E$ angiotensin-converting-enzyme, $A C S$ acute coronary syndrome, AT-II Angiotensine-II, $C A B G$ coronary artery bypass grafting, $C A D$ coronary artery disease, $C O P D$ chronic obstructive pulmonary disease. eGFR MDRD: estimated glomerular filtration rate according to the modification of diet in renal disease formula, CVA cerebral vascular accident, TIA transient ischaemic attack, $L M W H$ low-molecular-weight heparin, $M I$ myocardial infarction, $N$ number of patients, NYHA New York Heart Association functional classification, (N)OAC (non-)vitamin K antagonist oral anticoagulant, $P C I$ percutaneous coronary intervention, $S D$ standard deviation 
Table 3 Management of antiplatelet therapy in the pilot study
Fig. 3 Number of days ASA was discontinued preoperatively in patients on ASA monotherapy and the number of days clopidogrel and ticagrelor were discontinued in patients on DAPT. ASA acetylsalicylic acid, $D A P T$ dual antiplatelet therapy

\begin{tabular}{|c|c|c|c|}
\hline & ASA monotherapy & DAPT clopidogrel & DAPT ticagrelor \\
\hline \multicolumn{4}{|l|}{ Preoperative } \\
\hline Continued & 21 & 1 & 6 \\
\hline Discontinued & 20 & 4 & 13 \\
\hline $\begin{array}{l}\text { Days discontinued, median } \\
\text { (IQR) }\end{array}$ & $6(2)$ & $6(3.5)$ & $5(5.5)$ \\
\hline \multicolumn{4}{|l|}{ Postoperative } \\
\hline No restart & 0 & 2 & 10 \\
\hline Restart & 20 & 2 & 3 \\
\hline $\begin{array}{l}\text { Days after CABG until } \\
\text { restart, median (IQR) }\end{array}$ & $1(0)$ & $2.5(3)$ & $1(2)$ \\
\hline
\end{tabular}

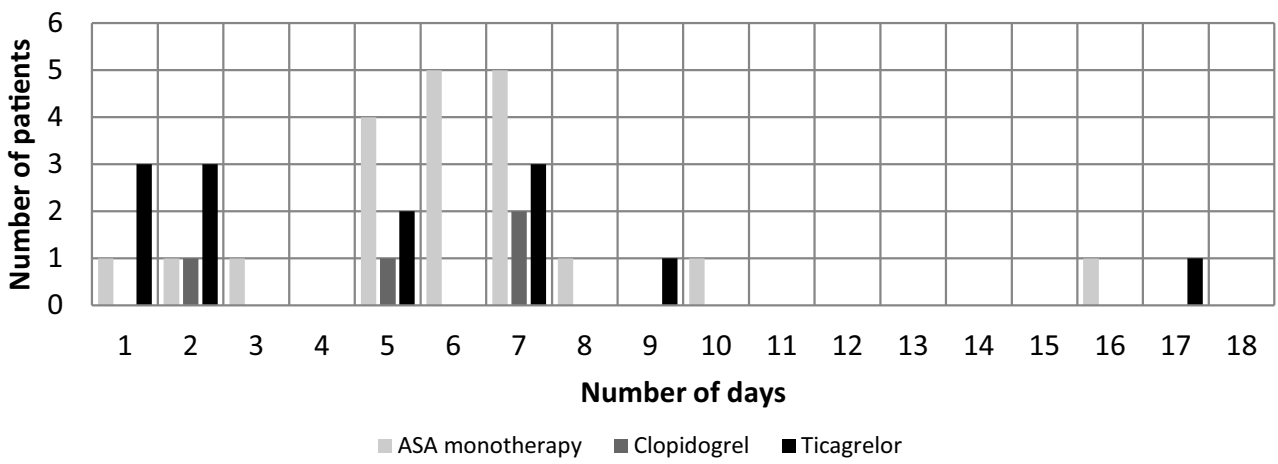

In the 4 centres in which ASA treatment was discontinued before surgery, one centre discontinued ASA 5 days before surgery, a second centre 4 days, a third centre 3 days and the fourth centre 2 days prior to surgery. This discrepancy in the time of discontinuation of treatment also applied to the management of $\mathrm{P} 2 \mathrm{Y}_{12}$ inhibitor use (Table 1).

\section{Registry}

A total of 70 patients underwent isolated CABG in the St. Antonius Hospital in Nieuwegein in July 2014 and were included in this pilot study. Baseline data are presented in Table 2. Of these 70 patients, 41 were on ASA monotherapy, 28 used a $\mathrm{P}_{2} \mathrm{Y}_{12}$ inhibitor and 1 patient was on ASA plus acenocoumarol treatment before surgery. From the 28 patients using a $\mathrm{P}_{2} \mathrm{Y}_{12}$ inhibitor, 9 were on clopidogrel and 19 were on ticagrelor.

Table 3 shows the preoperative management for patients treated with ASA monotherapy, for patients using clopidogrel as part of DAPT treatment and for patients using ticagrelor as part of DAPT treatment. Of the total of 70 patients, 2 were on clopidogrel due to intolerance for ASA. One of them continued to use clopidogrel. One patient was treated with triple therapy (ASA/clopidogrel/ acenocoumarol) and the last patient was treated with clopidogrel and acenocoumarol. The patient on triple therapy continued the acenocoumarol and stopped ASA and clopidogrel. The patient on acenocoumarol plus clopidogrel treatment continued the acenocoumarol and stopped the clopidogrel. Fig. 3 shows the number of days that medication was discontinued preoperatively. In the group of patients who discontinued ticagrelor, 8 patients had experienced an ACS less than 1 month before surgery. In the group that continued ticagrelor, 5 patients had experienced an ACS within 1 month before surgery.

After surgery, 68 out of 70 patients received ASA. Treatment was started the day after surgery in all patients. All these patients received a loading dose of $500 \mathrm{mg}$ intravenously. The two patients who did not receive ASA postoperatively were both preoperatively treated with clopidogrel monotherapy due to ASA intolerance. Both these patients received their first doses of clopidogrel the day after surgery.

Fig. 4 shows the postoperative management of $\mathrm{P} 2 \mathrm{Y}_{12}$ inhibitors. Patients who received a $\mathrm{P}_{2} \mathrm{Y}_{12}$ inhibitor after CABG did not receive a loading dose, but a regular maintenance dose. There was no apparent relationship between restarting DAPT postoperatively and a preoperative history of ACS or percutaneous coronary intervention (PCI) with stent implantation. The incidence of postoperative complications within 30 days was low. Myocardial infarction (MI), stroke and death were not observed, while Bleeding Aca- 
a

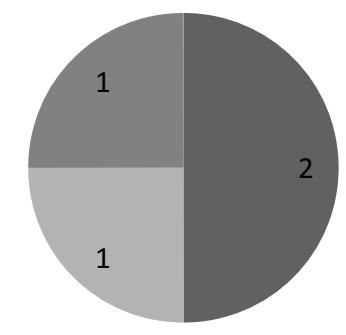

- First doses after $>24$ hours

First doses after $<24$ hours

Did not restart b

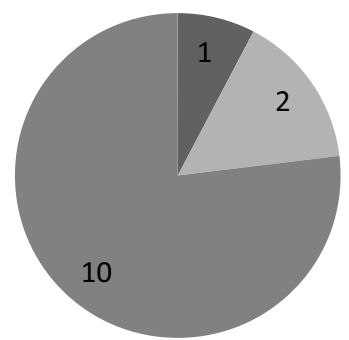

First doses after $>24$ hours

First doses after $<24$ hours

Did not restart

Fig. 4a,b Postoperative management of clopidogrel and ticagrelor in patients preoperatively on DAPT. DAPT dual antiplatelet therapy

demic Research Consortium (BARC) type 4 major bleeding occurred in 3 patients and 2 patients needed surgery for mediastinitis. Due to the small study population with subgroups and low incidence of postoperative complications, we decided not to analyse these postoperative complications in more detail.

\section{Discussion}

The results from this national survey regarding the perioperative management of antiplatelet treatment in CABG patients show major variability across the different Dutch centres. This variability partly reflects the disparity in recommendations in the different international guidelines [1-4]. A survey regarding antithrombotic treatment in CABG patients conducted in 1989 also showed major differences in antithrombotic treatment between Dutch cardiothoracic centres [7]. Although some treatment strategies that were used at the time (coumarins and dipyridamole) have since been abandoned for routine use in CABG patients, the variability in treatment strategies persists.

\section{CABG in patients using ASA monotherapy}

The management of patients with ASA monotherapy differs greatly between centres, both in continuing or discontinuing ASA before surgery and in the timing of discontinuation. Although it might be reasonable to stop ASA in patients with the highest bleeding risk [3, 4], the guidelines do not support discontinuation of ASA in the majority of patients, contrary to what appears to be the standard in many cardiothoracic centres who responded to this survey. The differences regarding the preoperative discontinuation of ASA in the guidelines and routine treatment in these cen-

tres might be caused by a lack of convincing evidence from randomised clinical trials. A recent meta-analysis including a total of 2399 patients showed that ASA exposure within 7 days before CABG, with or without concomitant surgery, resulted in a $44 \%$ reduction in the odds of MI [8]. However, it also resulted in a dose-dependent increase in blood loss, an increased volume of red cell transfusion and rate of surgical re-exploration without an effect on mortality.

In the largest randomised clinical trial to date regarding the preoperative use of ASA in CABG patients $(n=2100)$, [9] no differences were observed between patients using ASA or placebo with regard to any of the primary outcomes, death, MI, stroke, renal failure, pulmonary embolism and bowel infarction at 30 days after surgery (RR $0.9495 \%$ CI $0.80-1.12$ ). There were also no significant differences in the number of reoperations for bleeding or cardiac tamponade. Therefore, there is no clear benefit of ASA treatment before CABG.

The postoperative use of ASA in CABG patients is recommended in all guidelines as it has been proven to increase venous graft patency and reduces the occurrence of ischaemic events during follow-up in all patients regardless of revascularisation strategy [10-13].

\section{CABG in patients using DAPT}

Our survey revealed that the discrepancies in treatment strategies between the different centres were even greater in patients who received DAPT. Centres also differed in postoperative antiplatelet management, but the majority of centres stop the $\mathrm{P} 2 \mathrm{Y}_{12}$ inhibitor without restarting it after surgery. The timing of discontinuation of the $\mathrm{P} 2 \mathrm{Y}_{12}$ inhibitor varied roughly from 4 to 10 days between centres. A recent study from Hansson et al. shows that it is safe to discontinue ticagrelor 3 days and clopidogrel 5 days prior to CABG [14]. Many guidelines mention the option of preoperative bridging therapy with small molecule GPIIb/ IIIa inhibitors (i.e. eptifibatide or tirofiban) or cangrelor after discontinuation of $\mathrm{P} 2 \mathrm{Y}_{12}$ inhibitors in patients with an increased risk for ischaemic events (e.g. with recently implanted drug-eluting stent (DES)), but there is still little clinical evidence for this strategy. None of the respondents mentioned the use of this strategy in their centre.

Not restarting the $\mathrm{P} 2 \mathrm{Y}_{12}$ inhibitor after surgery is not supported by the guidelines, which recommend restarting DAPT after CABG as soon as it is considered safe and to continue DAPT for at least 12 months following ACS (class I, level A) [3, 15]. The ESC guidelines on revascularisation and non-ST-segment elevation ACS (NSTE-ACS) were updated after we received answers for our survey, but the 2011 ESC guideline on NSTE-ACS also stated that ticagrelor or clopidogrel should be considered to be (re-)started after CABG surgery as soon as considered safe (class IIa, 
level B) [16]. The recommendations from these guidelines are based on sub-analyses from three large randomised trials in ACS patients: the Clopidogrel in Unstable Angina to Prevent Recurrent Events (CURE) study [17], the TRial to Assess Improvement in Therapeutic Outcomes by Optimizing Platelet InhibitioN with Prasugrel-Thrombolysis In Myocardial Infarction (TRITON-TIMI 38) and the PLATelet inhibition and patient Outcomes (PLATO) study [18, 19]. These three trials all showed some benefit of continuation of DAPT after CABG in sub-analyses. However, the trials are underpowered and these post-hoc analyses have many limitations. The reason for the discontinuation of study treatment after surgery in a substantial number of patients is not reported in these trials, so there might be a selection bias. Furthermore, the percentage of patients who underwent CABG was relatively low (16.5\% in CURE, $2.5 \%$ in TRITON-TIMI 38, and 6.8\% in PLATO).

There is still much uncertainty as to how $\mathrm{P}_{2} \mathrm{Y}_{12}$ inhibitor treatment improves clinical outcome in this group and which patients should receive it at which particular moment. Outcomes might be improved due to an increase in vein graft patency with the use of a $\mathrm{P} 2 \mathrm{Y}_{12}$ inhibitor, as vein graft occlusion occurs in up to $26 \%$ of grafts after 1 year in patients using ASA monotherapy [20]. Multiple studies investigating the routine use of $\mathrm{P}_{2} \mathrm{Y}_{12}$ inhibitors in CABG patients are currently recruiting patients, including the The Effect Of Ticagrelor On Saphenous Vein Graft Patency In Patients Undergoing Coronary Artery Bypass Grafting Surgery (POPular CABG) study (clinicaltrials.gov identifier NCT02352402) and the Study Comparing Ticagrelor With Aspirin for Prevention of Vascular Events in Patients Undergoing CABG (TiCAB) study (clinicaltrials.gov identifier NCT01755520). Data from these studies might help us better assess the benefits and risks of antiplatelet therapy in all patients undergoing $\mathrm{CABG}$.

\section{CABG after PCI without prior ACS}

For patients undergoing $\mathrm{CABG}$ after PCI without prior ACS it is recommended to continue DAPT for at least 1 month after implantation of a bare metal stent (BMS) (class I, level A) and at least 6 months after a new-generation DES (class I, level B )[4]. However, the guidelines do not specify which postoperative therapy is advised if the target vessel has been bypassed.

The guidelines offer different options for the timing of both preoperative discontinuation and postoperative re-initiation of $\mathrm{P} 2 \mathrm{Y}_{12}$ inhibitor treatment. It should be considered to withhold clopidogrel and ticagrelor for 5 days and prasugrel for 7 days prior to surgery (class IIA, level C) [1-4]. Postoperatively, DAPT should be restarted within $24 \mathrm{~h}$ if it is deemed safe, with a loading dose of the $\mathrm{P}_{2} \mathrm{Y}_{12}$ inhibitor to optimise vein graft patency (Class IIA, level C) [3, 4]. The guideline from the American College of Chest Physicians specifies that when CABG is performed less than 6 weeks after BMS or less than 6 months after DES, DAPT should be continued during surgery to prevent stent thrombosis (Grade 2C) [2].

\section{Registry results}

The results of our registry in the St. Antonius Hospital demonstrate that there are major differences even in a single centre. Generally, the $\mathrm{P}_{2} \mathrm{Y}_{12}$ inhibitor is discontinued for a shorter time period before surgery than is advised by the guidelines. The reasons for this could be that patients were considered to be at higher risk for ischaemic events.

\section{Limitations}

Multiple limitations regarding the survey and registry merit mention. A questionnaire will only result in a general depiction of clinical practice, although we tried to include open questions to gather information regarding treatment of patients who did not fall into standard treatment protocols. However, treatment might actually differ from the answers provided by the responders as individual physicians might deviate from local protocols. Furthermore, only $69 \%$ of centres responded to our questionnaire.

The pilot study is limited due to its single-centre nature and the small population size. Another limitation for both studies is that the new ESC guideline regarding non-STsegment-elevation myocardial infarction was published after the survey and the registry were conducted. Adherence to the guidelines might have increased since.

\section{Conclusion}

Dutch cardiothoracic centres are not unified in their perioperative management of antiplatelet therapy in patients undergoing isolated CABG. The lack of evidence from randomised controlled trials could contribute to these differences between centres. More evidence from ongoing trials is essential to better evaluate the benefits and risks of antiplatelet therapy in $\mathrm{CABG}$ patients and strengthen the recommendations of the guidelines.

Acknowledgements We would like to thank the following people for their participation in our survey: Dr. M. P. Buijsrogge from University Medical Center Utrecht, Dr. B. Martina from Leiden University Medical Center, Prof. A. P. Kappetein from the Erasmus Medical Center, in Rotterdam, Drs. W. W. L. Li from the Academic Medical Centre in Amsterdam, Drs. A. Tjon from the Onze Lieve Vrouwe Gasthuis in Amsterdam, Drs. B. Maesen from Maastricht University Medical Center+, Drs. C. A. Boot from Amphia Hospital in Breda, Drs. N. J. Verberkmoes from Catharina Hospital in Eindhoven, Mr. M. M. Bos from Medisch Spectrum Twente in Enschede, and Prof. P. W. Boonstra Medical Centre Leeuwarden. 
Funding The Department of Cardiology in the St. Antonius Hospital receives funding from AstraZeneca for the POPular CABG study, which is a randomised clinical trial involving ticagrelor in CABG patients. The authors did not receive any funding for the work described in this article.

Conflict of interest P.W.A. Janssen, D.M.F. Claassens, L.M. Willemsen, T.O. Bergmeijer and P. Klein declare that they have no competing interests. J. M. ten Berg reports receiving advisory/consulting/speakers fees from AstraZeneca, Eli Lilly, Daiichi Sankyo, the Medicines Company, Accumetrics, Boehringer-Ingelheim, BMS, Pfizer, Bayer.

Open Access This article is distributed under the terms of the Creative Commons Attribution 4.0 International License (http:// creativecommons.org/licenses/by/4.0/), which permits unrestricted use, distribution, and reproduction in any medium, provided you give appropriate credit to the original author(s) and the source, provide a link to the Creative Commons license, and indicate if changes were made.

\section{References}

1. Hillis LD, Smith PK, Anderson JL, et al. ACCF/AHA Guideline for Coronary Artery Bypass Graft Surgery. A report of the American College of Cardiology Foundation/American Heart Association Task Force on Practice Guidelines. Circulation. 2011;124:2610-42.

2. Douketis JD, Spyropoulos AC, Spencer FA, et al. Perioperative management of antithrombotic therapy: antithrombotic therapy and prevention of thrombosis, 9th ed: American College of Chest Physicians Evidence-Based Clinical Practice Guidelines. Chest. 2012;141(2 Suppl):e326S-e50S.

3. Ferraris VA, Saha SP, Oestreich JH, et al. Update to the Society of Thoracic Surgeons guideline on use of antiplatelet drugs in patients having cardiac and noncardiac operations. Ann Thorac Surg. 2012;94:1761-81.

4. Kolh P, Windecker S, Alfonso F, et al. ESC/EACTS Guidelines on myocardial revascularization: the Task Force on Myocardial Revascularization of the European Society of Cardiology (ESC) and the European Association for Cardio-Thoracic Surgery (EACTS). Developed with the special contribution of the European Association of Percutaneous Cardiovascular Interventions (EAPCI). Eur J Cardiothorac Surg. 2014;46:517-92.

5. Steg PG, James SK, Atar D, et al. ESC Guidelines for the management of acute myocardial infarction in patients presenting with STsegment elevation. Eur Heart J. 2012;33:2569-619.

6. Krimly A, Yan RT, Yan AT, et al. Use of clopidogrel post-coronary artery bypass surgery in canadian patients with acute coronary syndromes. Can J Cardiol. 2011;27:711-5.

7. Galema TW, Verheugt FWA. Antithrombotica bij coronaire bypassoperaties in Nederland. Ned Tijdschr Geneeskd. 1990;134:1632-6.
8. Hastings S, Myles P, McIlroy D. Aspirin and coronary artery surgery: a systematic review and meta-analysis. $\mathrm{Br} \mathrm{J}$ Anaesth. 2015;115:376-85.

9. Myles PS, Smith JA, Forbes A, et al. Stopping vs. continuing aspirin before coronary artery surgery. N Engl J Med. 2016;374:728-37.

10. Lorenz RL, Schacky CV, Weber M, et al. Improved aortocoronary bypass patency by low-dose aspirin (100 mg daily). Effects on platelet aggregation and thromboxane formation. Lancet. 1984;1(8389):1261-4.

11. Fuster V, Chesebro JH. Role of platelets and platelet inhibitors in aortocoronary artery vein-graft disease. Circulation. 1986;73: 227-32.

12. Goldman S, Copeland J, Moritz T, et al. Improvement in early saphenous vein graft patency after coronary artery bypass surgery with antiplatelet therapy: results of a Veterans Administration Cooperative Study. Circulation. 1988;77:1324-32.

13. Gavaghan TP, Gebski V, Baron DW. Immediate postoperative aspirin improves vein graft patency early and late after coronary artery bypass graft surgery. A placebo-controlled, randomized study. Circulation. 1991;83:1526-33.

14. Hansson EC, Jidéus L, Åberg B, et al. Coronary artery bypass grafting-related bleeding complications in patients treated with ticagrelor or clopidogrel: a nationwide study. Eur Heart J. 2016;37:189-97.

15. Roffi M, Patrono C, Collet JP, et al. ESC Guidelines for the management of acute coronary syndromes in patients presenting without persistent ST-segment elevation: Task Force for the Management of Acute Coronary Syndromes in Patients Presenting without Persistent ST-Segment Elevation of the European Society of Cardiology (ESC). Eur Heart J. 2015;37:267-315.

16. Hamm CW, Bassand JP, Agewall S, et al. ESC Guidelines for the management of acute coronary syndromes in patients presenting without persistent ST-segment elevation: The Task Force for the management of acute coronary syndromes (ACS) in patients presenting without persistent ST-segment elevation of the European Society of Cardiology (ESC). Eur Heart J. 2011;32:2999-3054.

17. Yusuf S, Zhao F, Mehta SR, et al. Effects of clopidogrel in addition to aspirin in patients with acute coronary syndromes without STsegment elevation. N Engl J Med. 2001;345:494-502.

18. Wallentin L, Becker RC, Budaj A, et al. Ticagrelor versus clopidogrel in patients with acute coronary syndromes. N Engl J Med. 2009;361:1045-57.

19. Wiviott SD, Braunwald E, McCabe $\mathrm{CH}$, et al. Prasugrel versus clopidogrel in patients with acute coronary syndromes. N Engl J Med. 2007;357:2001-15.

20. Alexander JH, Hafley G, Harrington RA, et al. Efficacy and safety of edifoligide, an E2F transcription factor decoy, for prevention of vein graft failure following coronary artery bypass graft surgery: PREVENT IV: a randomized controlled trial. JAMA. 2005;294:2446-54. 